\begin{tabular}{|c|c|c|c|}
\hline Eiszeitalter u. Gegenwart & 43 & $101-109$ & Hannover 1993 \\
\hline
\end{tabular}

\title{
Renaturierung von Sand- und Kiesgruben
}

\author{
Jens Dieter Becker-Platen *)
}

Lagerstätten, Abbau, Renaturierung

\begin{abstract}
Kurzfassung: Mineralische Rohstoffe werden in großen Mengen für die verschiedensten Wirtschaftszweige benötigt. Die Gewinnung oberflächennaher Rohstoffe bedingt i. a. eine Abbaugrube, welche später wieder in die Umgebung eingefügt, d. h. rekultiviert oder renaturiert, werden muß. Im Zuge eines erstarkenden Umwelt- und Ökologiebewußtseins ist der Renaturierung verstärkte Aufmerksamkeit zu schenken.
\end{abstract}

\section{[Leaving to natural revegetation of sand- and gravelpits]}

\begin{abstract}
Mineral resources are required for different sections of the economy. Working resources cloth to the surface generally causes a pit, which later on must be integrated into the surrounding landscape, that is it must be recultivated or left to natural revegetation.
\end{abstract}

\section{Rohstoffgewinnung}

Die Gewinnung und Nutzung mineralischer Rohstoffe ist so alt wie die Menschheitsgeschichte selbst. Sie ist heute mehr denn je eine unabdingbare Voraussetzung für die Wirtschaft einer Industrienation, daher werden jährlich riesige Mengen an Rohstoffen gewonnen und verbraucht. So ist die Bundesrepublik Deutschland einer der größten Rohstoffkonsumenten der Welt; z. B. der Welt zweitgrößter und Europas größter Kiesverbraucher.

Unser Rohstoffbedarf wird verursacht durch die Lebens- und Verbrauchsgewohnheiten jedes einzelnen Bürgers, auch jener, die vehement gegen Steinbrüche und Kiesgruben lamentieren, und er wird durch ein Abbauverbot, wie es teilweise für ausgewählte Rohstoffe in bestimmten Gebieten gefordert wird, nicht gesenkt, sondern nur in andere Räume verlagert (St.-Florians-Prinzip). Allein an Sand und Kies wurden in Niedersachsen und Bremen 1990 fast 50 Mio. $t$ verbraucht, das sind rund $7 \mathrm{t} /$ Pers. im Jahr, und Niedersachsen steht innerhalb der Bundesrepublik hier nicht in vorderster Reihe.

\footnotetext{
*) Anschrift des Autors: Vizepräsident und Professor der Bundesanstalt für Geowissenschaften und Rohstoffe und des Niedersächsischen Landesamtes für Bodenforschung, Dr. J. D. Becker-Platen, Stilleweg 2, 30655 Hannover
}

Es gibt vielfältige Bestrebungen, diesen hohen Rohstoffverbrauch zu senken, z. B. durch Wiederverwendung bereits genutzter Rohstoffe (Recycling) oder durch Ersatz durch andere Stoffe (Substitution). Aber allen diesen Bestrebungen sind enge Grenzen gesetzt. Bis auf wenige Ausnahmen wird nur ein relativ kleiner Teil der natürlichen Rohstoffe ersetzbar sein, wie am Beispiel des Bauschutt- und Straßenaufbruchrecyclings in Niedersachsen deutlich erkennbar ist. Hier fielen 1987 2,3 Mio. t Bauschuttund 0,9 Mio. t Straßenaufbruchmaterialien an. Dies sind 6,5\% des jährlichen Sand- und Kiesverbrauchs (knapp 50 Mio. t in 1990). Mehr könnte durch diese Recyclingprodukte also gar nicht ersetzt werden, selbst wenn sie zu $100 \%$ wiederverwendet würden, was aber nicht erreichbar ist.

\section{Naturschutz und Bodenabbau}

Die Gewinnung oberflächennaher Rohstoffe bedeutet stets einen mehr oder weniger nachhaltigen Eingriff in unsere fast ausnahmslos vom Menschen geprägte Kulturlandschaft. In der Regel findet jedoch nur eine Zwischennutzung von einigen Jahren bis Jahrzehnten statt. Städte und Dörfer dagegen bleiben uns Jahrhunderte, forstliche Monokulturen, kanalisierte Fließgewässer und ausgeräumte Landschaften viele Jahrzehnte erhalten.

Seit Ende 1976 ist das Bundesnaturschutzgesetz als Rahmengesetz in Kraft und seitdem mehrfach novelliert worden, die neueste Änderung ist gerade im Verfahren. Die einzelnen Länder haben auf dieser Grundlage inzwischen eigene gesetzliche Regelungen getroffen, die - im Hinblick auf die Steine- und Erden-Industrie - insgesamt zu restriktiveren Genehmigungsverfahren, zu mehr naturbezogenen Wiederherrichtungs- bis hin zu Renaturierungsauflagen geführt haben.

Seit langem ist also zu erkennen, daß versucht wird, einen fast "ungehemmten", ungeregelten Bodenabbau, wie er - zugegebenermaßen - von den 50er bis Anfang der 70er Jahre nicht selten durchgeführt wurde, mehr und mehr mittels durchaus berechtigter und sinnvoller gesetzlicher Regelungen einzu- 


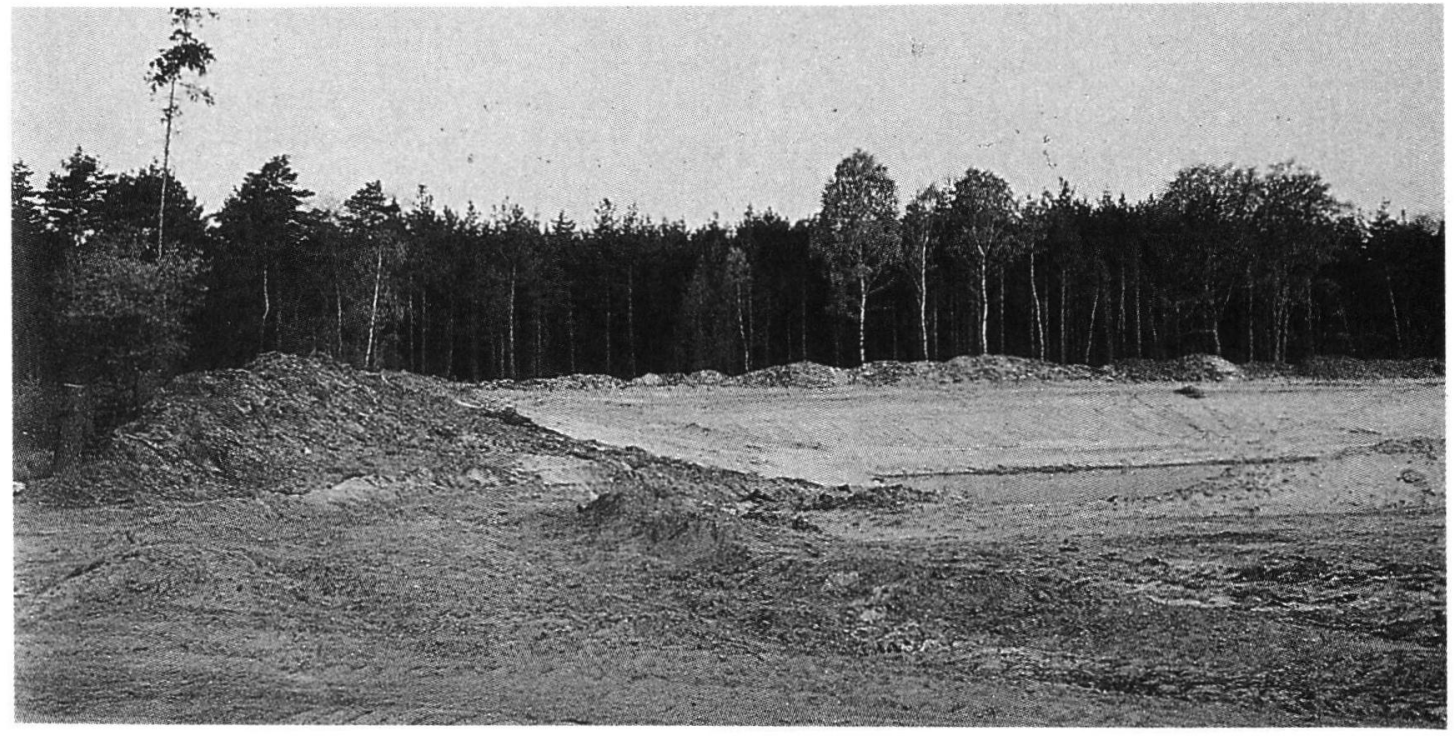

Abb. 1: Ausgebeutete Sandgrube mit wenig Rekultivierungsarbeiten

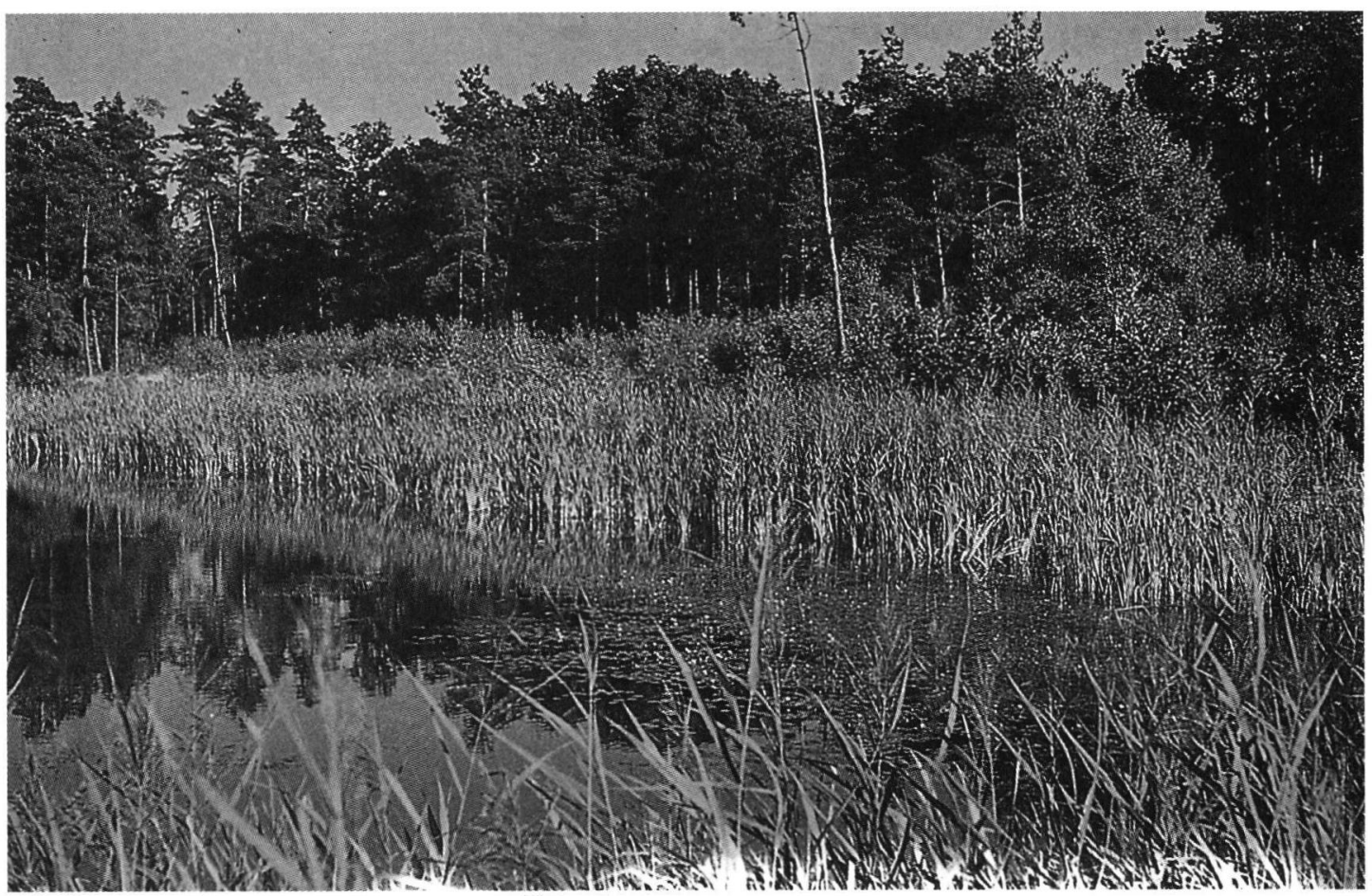

Abb. 2: ... und einige Jahre danach, die Grube hat sich renaturiert

schränken. Aber wir müssen auch zur Kenntnis nehmen, daß es immer schwieriger wird, der Volkswirtschaft die benötigten heimischen Rohstoffe kostengünstig, d. h. zu volkswirtschaftlich vertretbaren Bedingungen, bereitzustellen, weil u. a. die Ansprüche des Natur- und Umwelt- sowie des Grundwasser- schutzes inzwischen politisch einen sehr hohen Stellenwert haben und daher häufig höher bewertet werden als die Sicherung heimischer Rohstoffe. Dies ist in allen Bundesländern zu erkennen.

Ein jüngstes Beispiel aus Niedersachsen sei hierfür angeführt. Dort wird zur Zeit das Landes-Raumord- 
nungsprogramm "unter ökologischen Gesichtspunkten" fortgeschrieben, so, wie es die Koalitionsvereinbarung vom Juni 1990 fordert. Im Entwurf des neuen Programms heißt es daher auch folgerichtig: "... Abbauvorhaben sollen so durchgeführt werden, daß die ökologische und gestalterische Wiedereingliederung der Abbaufläche in die Landschaft beschleunigt wird ..."

Und an anderer Stelle wird ausgeführt:

"... Sofern Gebiete nicht mehr landwirtschaftlich, durch Bodenabbau oder sonstige Inanspruchnahme genutzt werden, ist sicherzustellen, daß darin Lebensräume für die heimische Tier- und Pflanzenwelt geschaffen werden. Dies gilt insbesondere für die Bodenabbaugebiete."

Dies ist eine sehr weitgehende Forderung, weil ja viele Flächeneigentümer nach einem Abbau durchaus auch andere Nutzungen anstreben mögen, eben nicht nur eine Naturschutz-Folgenutzung, und daher werden sie unter Umständen ihre Flächen bei den neuen Vorgaben für eine Gewinnung mineralischer Rohstoffe gar nicht erst zur Verfügung stellen. Hier müssen die nächsten Jahre zeigen, wie strikt diese Absichten denn auch tatsächlich umgesetzt werden sollen oder können.

Nicht unerwähnt bleiben darf hier die Tatsache, daß schon in der Vergangenheit und auch heute vielerorts ehemalige Abbauflächen durchaus freiwillig oder aber aufgrund von Auflagen im Genehmigungsverfahren renaturiert, d. h. Naturschutzzwecken zugeführt wurden bzw. werden.

Es gibt inzwischen eine Vielzahl qualifizierter Studien über den Naturschutzwert stillgelegter, sich selbst überlassener Abbaustellen. In aller Regel beschreiben sie ein erfreulich reichhaltiges Artenspektrum für Flora und/oder Fauna. Insbesondere Tongruben, Kalk- und Gipssteinbrüchen galt in der Vergangenheit das Interesse. Bei Sand- und Kiesgruben aber besteht hier noch ein erheblicher Nachholbedarf. Zumeist wurde nur die Avifauna von Baggerseen studiert, während andere Tier- und Pflanzengruppen, insbesondere im Bereich der Trockenabbauflächen, weitgehend vernachlässigt wurden.

\section{Rekultivierung}

In der Vergangenheit wurden ehemalige Abbaustellen häufig land- oder forstwirtschaftlich genutzt, als Deponie verfüllt (oft genug unsere heutigen Altlasten) oder auch als begehrtes Erholungsgelände genutzt, z. B. als Badesee in Anspruch genommen.

Dies war seinerzeit die Folge des ästhetischen Empfindens vieler Mitbürger, aufgrund dessen "unansehnliche, offene Löcher", dazu noch fast beliebig in der Gegend verteilt, nicht in unsere typisch deut- sche, aufgeräumte und durchplante Landschaft paßten. Die Gruben mußten rekultiviert werden, damit sie der Vorstellung von einer "ordentlichen" Landschaft, möglichst der flurbereinigten Umgebung des Wohnortes entsprachen.

Heute wissen wir, daß diese Art der Rekultivierung der Natur vielfach mehr schadete als nutzte, auch wenn sie zu ihrer Zeit nach Stand von Wissenschaft und Technik durchgeführt wurde und sicher damals z. T. auch ihre Berechtigung hatte.

\section{Renaturierung}

Manchmal aber überließ man, wie bereits aufgeführt, stillgelegte Gruben oder Brüche sich selbst. Dadurch verblieben dort in großen Bereichen "unbehandelte", unaufgeräumte, nicht rekultivierte Flächen, auf denen der nackte Kalkstein, Sand, Ton oder auch andere Gesteine anstanden. Diese Rohböden waren und sind Standorte für Pioniere, für Erstbesiedler aus Fauna und Flora. Oft sind es Standorte mit extremen Lebensbedingungen, geeignet für ganz besondere Spezialisten und immer bieten sie ihnen Überlebenschancen und Entwicklungsmöglichkeiten, die andernorts in unserer weitgehend vergleichmäßigten Kunstlandschaft nur noch selten zu finden sind.

In solchen, unbewußt der Natur zur Eigenentwicklung überlassenen Abbaustellen entwickelten sich in einer Vielzahl von Fällen, wie im übrigen auf manchen anderen stark anthropogen geprägten Standorten auch (z. B. Schlammteiche, alte Bahntrassen), Biotope, welche aus heutiger Sicht erhaltenswert sind ("Biotope aus 2. Hand"), weswegen sie vielerorts auch bereits unter Naturschutz gestellt wurden. Derartige klein- und großflächige Biotope können helfen, ein Mosaik, eine Vernetzung von naturnahen Zellen als Rückzugs- und davon ausgehend als Regenerationsgebiete für bedrohte Arten von Fauna und Flora zu schaffen, also eine "Trittsteinfunktion" ausüben. Eine Vielzahl solcher untereinander und über verschiedene Landschaftsteile auch miteinander verknüpfter Lebensräume unterschiedlichster Ausprägung kann ein erfolgversprechender Schritt zur Schaffung vernetzter Strukturen sein.

Für Abbaubetreiber und für Naturschutzbehörden gemeinsam sollte künftig die Devise lauten: weniger Rekultivierung, weniger Einbindung, weniger Angleichung an die umgebende Kulturlandschaft, dafür aber mehr Einfühlung in die Selbstheilungskräfte der Natur und hierzu nur leichte Starthilfen geben.

Die Tatsache, daß durch die "Folgenutzung Naturschutz" eine Fläche der weiteren wirtschaftlichen Nutzung entzogen wird, muß dabei wohl oder übel hingenommen werden. Dies entspricht der heute 


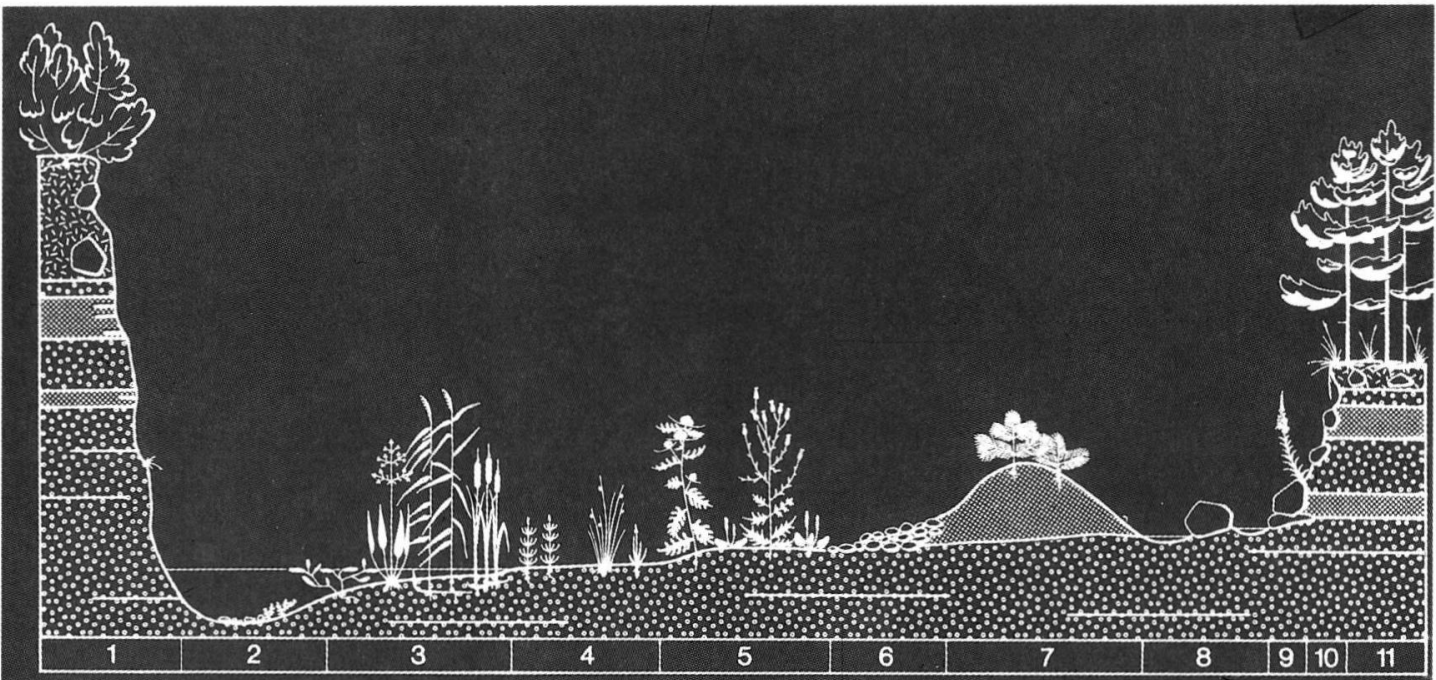

\section{Schnitt durch eine Kiesgrube mit verschiedenen Kleinbiotopen}

1. Steilwand mit Sandlinsen, 2. Baggenwelher mit Tiefenzone, 3. Flachufer mit Verlandungsvegetation, 4. Wechselfeuchte Uferzone, 5. Trockener Kiesboden mit Odlandvegetation, 6. Vegetationsloser Steinhaufen, 7. Trockener Sandhaufen, 8. Tumpel, 9. Gesteinsblocke (Findlinge), 10. Südexponierter Stellhang, 11. Trockener Fohrenwald.

Abb. 3: Schnitt durch eine Kiesgrube mit verschiedenen Kleinbiotopen (nach: Deutscher Bund für Vogelschutz, 1981)

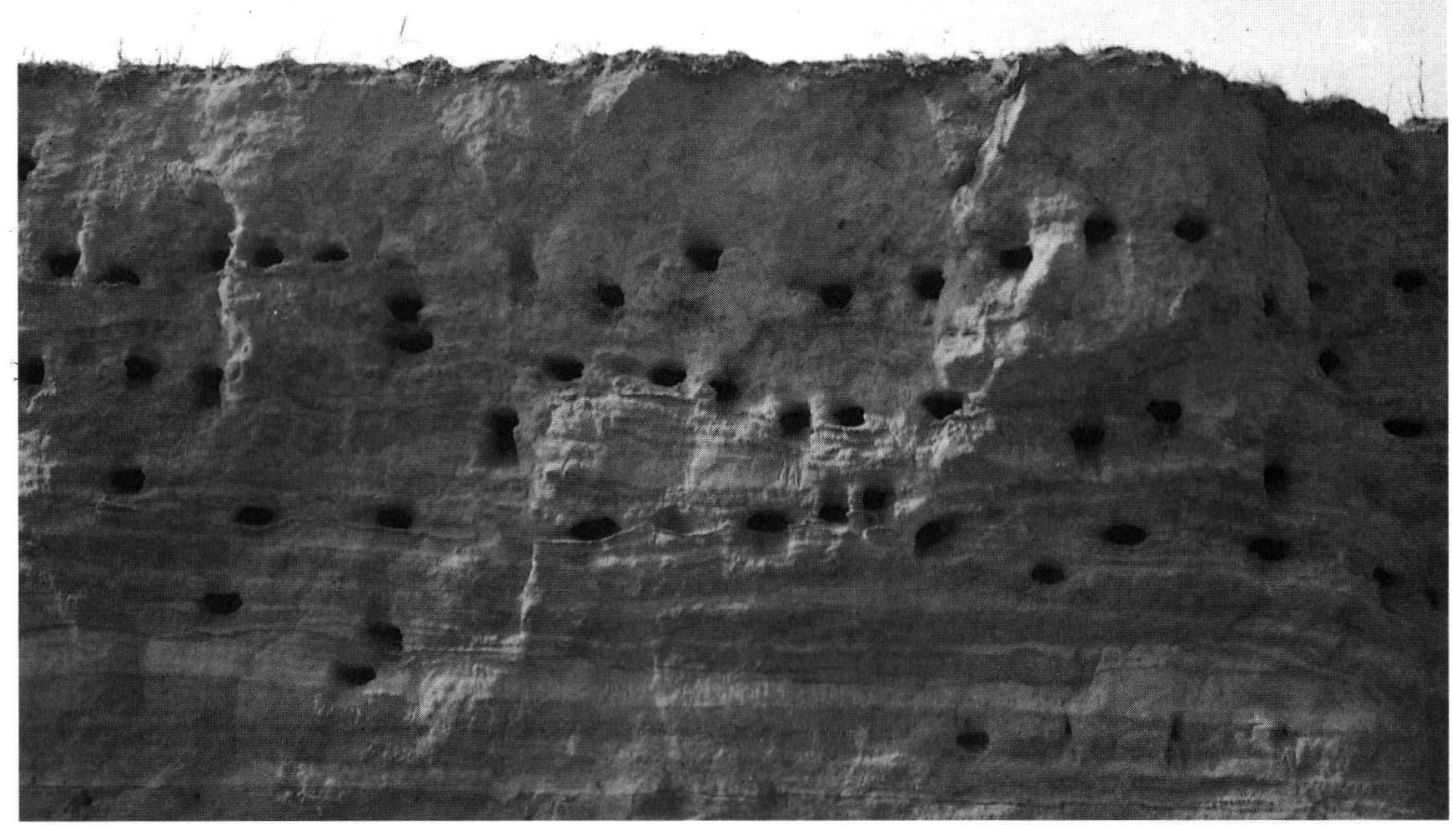

Abb. 4: Uferschwalben in Sandgrubenböschung 
weit verbreiteten gesellschaftlichen und politischen Grundhaltung zu diesen Dingen.

Inzwischen gibt es - neben einzelnen, leider immer noch unbelehrbaren Abbauunternehmern - erfreulicherweise eine große Zahl derer, die sich die Erkenntnisse der Renaturierungsmöglichkeiten zu eigen machen und in die Planung und Durchführung ihrer Abbauvorhaben einbringen. Es ist daher $\mathrm{zu}$ empfehlen und wird vielfach schon gesetzlich gefordert, möglichst frühzeitig die angestrebten Rekultivierungs- bzw. Renaturierungsziele einer Abbaustelle festzulegen. Hierdurch können u. a. die Abbauplanung und -technik sowie die Standortwahl für Verarbeitungsanlagen und Abraumdeponien optimiert werden.

\section{Renaturierung von Sand- und Kiesgruben}

Sand- und Kiesgruben sind die häufigsten Abbaustellen in unseren Landen. Bei kleineren Gruben ist eine Widmung für Naturschutzzwecke in aller Regel einfacher, da andere Nutzungsinteressenten weitgehend zurücktreten. Großflächige ehemalige Trocken- oder Naßabbauten aber erwecken wesentlich stärker die Begehrlichkeit z. B. von Land- und Forstwirtschaft oder der Freizeitnutzung, etwa als Moto-Cross-Piste oder Bade-/Campingsee. Zur Abwehr des Erholungsrummels ist eine möglichst früh- zeitige Naturschutzplanung mit entsprechenden betriebsbegleitenden Herrichtungen zu empfehlen, wie etwa einer reich strukturierten Kleinsttopographie von Sohle und Böschungen und die Abpflanzung des Grubenbereichs durch dornenbewehrte Sträucher, die zugleich mit ihren Früchten als Vogelnahrung dienen. Dornenhecken brauchen keine Pflege und werden im Laufe der Jahre "undurchdringlich". Dem Druck Erholungsuchender kann dabei zusätzlich durch weiträumige Absperrungen der Zufahrtswege (Dämme, Gräben, Schlagbäume) entgegengewirkt werden. Kaum ein Camping- oder Badegast läuft viele hundert Meter mit seiner gesamten Ausrüstung vom Autoparkplatz zum Ort des Vergnügens. Und wenn dort auch noch Haufen von Holz, Buschwerk, Steinen und Abraum den potentiellen Badestrand unansehnlich machen, ist die Lust am Baden und Campen meist vollends verflogen. Den stillen Wanderer hingegen stört dies nicht, vielmehr lernt er den Naturschutzwert solcher „unordentlicher" Gruben kennen und schätzen.

In Anlehnung an STEIN (1985) werden im folgenden einige Bedingungen genannt, die die Renaturierung von Sand- und Kiesgruben begünstigen:

\section{Trockenabbau}

- Mutterboden und Abraum sollten nicht gleichmäßig über die gesamte Grube incl. Böschungen

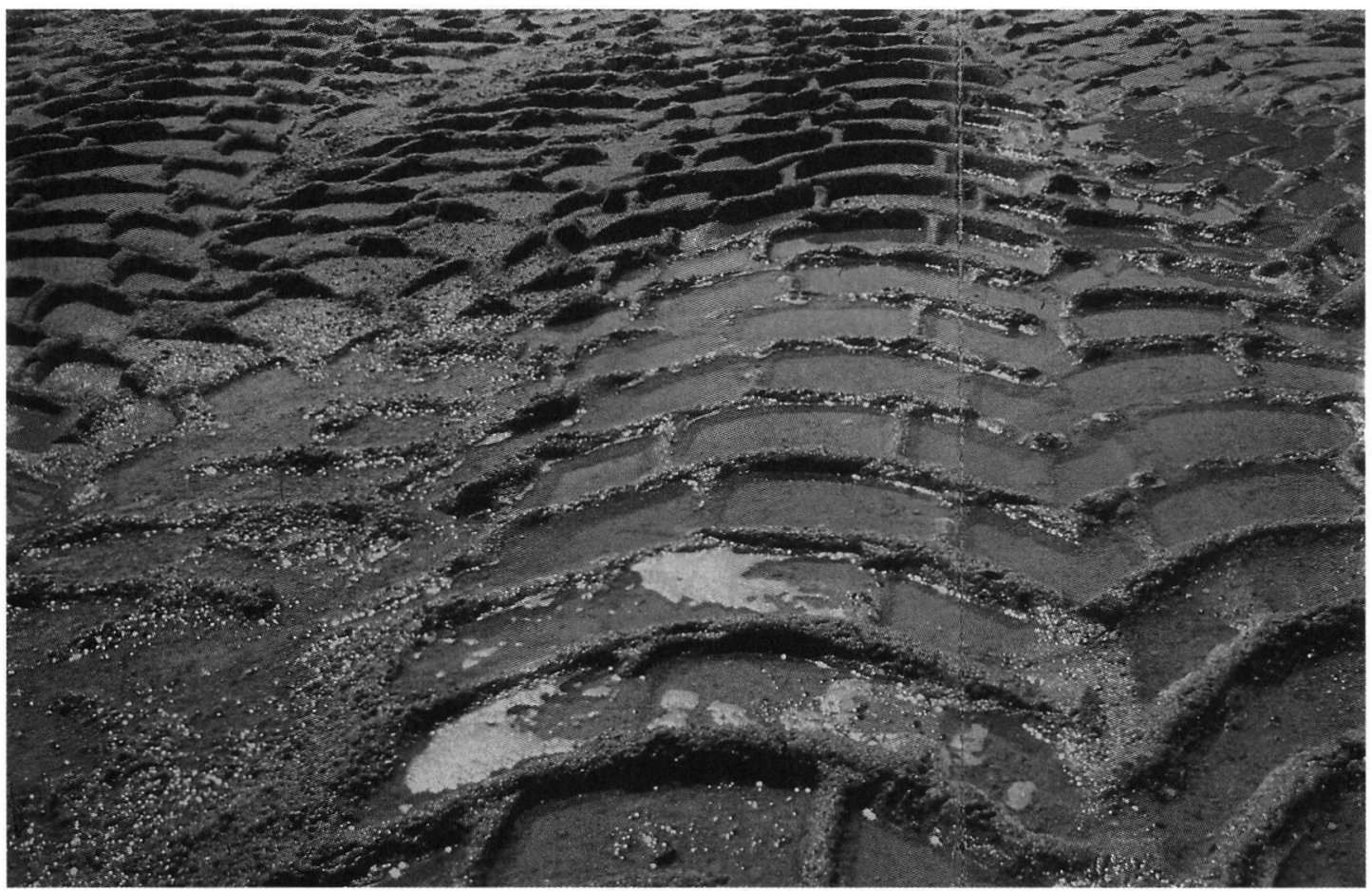

Abb. 5: Wassererfüllte Spuren von Radladerreifen in einer Sandgrube 
verteilt werden. Große nackte, möglichst vegetationsfreie Sand- und Kiesflächen (Rohböden) sind zu schaffen.

- Ökologisch wertvoll sind nicht abgedeckte südexponierte Böschungen und Steilwände.

- Ein gelegentliches Nachbrechen steiler Böschungen ist erwünscht, weil es günstig für die Neuanlagen der Niströhren von Uferschwalben etc. ist. Dabei ist ein ausreichender Abstand zu Bauwerken und zu Grundstücksgrenzen einzuplanen.

- Man sollte unterschiedlich tiefe, wassererfüllte Flächen, z. B. sich sammelndes Niederschlagswasser im Grubentiefsten oder einzelne Tümpel mit Grundwasseranschluß, schaffen. Beides ist hilfreich für das Entstehen versumpfender Bereiche und von Amphibienbiotopen.

- Tief eingefahrene Radladerspuren etc. sind nicht einzuebnen. Sie sind vielfach im Frühjahr die wassererfüllte Kinderstube zahlreicher Amphibien. Ein Austrocknen zu Beginn des Sommers ist unproblematisch, da Jungfrösche etc. sie dann bereits verlassen haben.

- Große Steine (Blöcke, Überkorn) sollten in Haufen zusammengeschoben werden, hier überwintern z. B. Amphibien und Reptilien, weiterhin sind sie Lebensraum für vielerlei Kleinsäuger.

- Reste von Abraum und unverkaufte Körnung sollten als Haufen auf der Grubensohle liegen bleiben. Sie sind ebenso wie die Böschungen teilweise extrem trockene Standorte und bieten vielen bodenbewohnenden Insekten ideale Lebensbedingungen.

- Baumstubben und Altholz sind zu Haufen zusammenzutragen; Flechten, Pilze und Insekten werden dadurch gefördert.

- Über das Grubengelände verstreut sind einzelne standortgerechte Gehölzgruppen anzupflanzen.

\section{$\mathrm{Naßabbau}$}

- Es sind weite, flache und vegetationsfreie Uferbereiche, ohne Abraum- oder Mutterbodenbedeckung zu schaffen; die Möglichkeit hierzu ist von den örtlichen Lagerstättengegebenheiten abhängig.

- Wenn der Grundwasserstand es erlaubt, sollten im Uferbereich und in der Flachwasserzone Haufen/Inseln aus gröberem Material (Überkorn, Blöcke) geschaffen werden (Brutplätze für Vögel).

- Durch Einbringen von Abraum oder inertem Bodenaushub und Bauschutt in begrenzten Berei- chen können Flachwasserzonen geschaffen werden. Allerdings wird durch Einbringen dieser im allgemeinen nährstoffreichen Substrate der See eutrophiert! Das Material ist nicht einzuplanieren; eine leicht wellig-kuppige Oberfläche erhöht die Standortvielfalt. Auch der Bereich des Spülfächers einer Kieswäsche oder ein Schlammteich sollten in Flachwasserzonen einbezogen werden.

- Breite treppenartig gestaltete Böschungen unter Wasser begünstigen durch unterschiedliche Wassertiefe die Pflanzenvielfalt.

- Schilfrohr-Stecklinge können in einigen Flachwasserbereichen eingebracht werden, sie breiten sich rasch aus.

- Weichholzgehölze sind im Uferbereich anzulegen (Weiden- und Pappelstecklinge).

- Nach Süden und Westen gerichtete Steilufer sollten nicht abgeschrägt werden, hier nisten Eisvogel und Uferschwalbe. Der Wellenschlag in der Wasserwechselzone (Bereich zwischen höchstem und niedrigstem Wasserstand) kann durch vorgeschaltete kleinere Böschungen gebrochen werden. Das Ufer wird im Laufe der Jahre durch Wellenschlag zurückverlegt, daher sind Sicherheitsabstände zu Bauwerken und Grundstücksgrenzen zu beachten.

- Es sollte kein Besatz mit Fischen und anderen Wassertieren erfolgen. Im Gefieder von Wasservögeln werden Fisch- und Froschlaich sowieso recht bald eingetragen.

- Durch Abtrennung kleinerer Buchten und "Lagunen" oder kleinerer separater Seebereiche kann eine Biotopvielfalt erreicht werden. Ebenso sollte die Uferlinie geschwungen und wechselweise mit Gebüsch und Bäumen oder auch ohne höheren Aufwuchs gestaltet werden.

Baggerseen, in welche Mutterboden und inerter Bodenaushub eingebracht wird und an deren Uferund Flachwasserzonen Laubgehölze bzw. Röhrichte gepflanzt werden, werden sich zu nährstoffreichen (eutrophen) Gewässern entwickeln. Sie sind für unsere an offenen Wasserflächen verarmte Landschaft zwar auch ein Gewinn, noch wichtiger aber, weil fast nicht mehr vorhanden, ist die Schaffung nährstoffarmer (oligothropher) Gewässer. Hierzu muß darauf geachtet werden, daß möglichst wenig Laubhölzer in der Umgebung des Sees stehen bzw. gepflanzt werden (Laub bedeutet Nährstoffeintrag in einen See). Gegen Düngereintrag durch den Wind aus angrenzenden landwirtschaftlichen Flächen schützt ein mit Büschen und Bäumen bewachsener Wall (möglichst hoher Nadelholzanteil). Durch ein Grabensystem um den Baggersee herum kann der Zufluß nährstoffreicher Oberflächenwässer aufgefangen und abgeleitet werden. 
Nährstoffhaltiges Grundwasser wird aber in aller Regel dennoch dem angestrebten oligothrophen See zufließen. Durch einen oberirdischen Seeabfluß/überlauf kann ein Teil davon mitsamt einer Menge organischen Materials (Algen etc.) wieder abgeführt werden. Bade- und Angelbetrieb verbietet sich an oligothrophen Seen.

Vom Naturschutz gefördert wird die Unterschutzstellung von großen Baggerseen oder auch von Anhäufungen kleinerer Gewässer in Flußniederungen. Sie dienen den Zugvögeln als Rastplätze, da die natürliche Flußlandschaft mit ihren gewundenen Fließgewässern und wassererfüllten Altarmen zumeist der Flußbegradigung und Flurbereinigung zum Opfer fiel.

Im folgenden seien einige besonders hervorzuhebende Renaturierungsziele in Sand- und Kiesgruben (Naß- und Trockenabbau) genannt:

Hart- und Weichholzauen wären von Natur aus in den Auen unserer Fließgewässer verbreitet. Diese aber werden heute überwiegend als Grünland, teilweise auch als Acker genutzt. Vereinzelt aber, mehr durch Zufall, fand die Natur bei der Wiederinbesitznahme alter Baggergruben Verhältnisse vor, die den Wiederaufwuchs eines Weichholz-Auenwaldes ermöglichten. Diese Gruben zeigen deut- lich schwankende Grundwasserstände und liegen i. a. im Überschwemmungsbereich eines nahen Fließgewässers. Teile der Gruben sind häufig bis knapp über den mittleren Grundwasserstand mit Abraum verfüllt, Böschungen z. T. abgeflacht und als Initialzündung wurden ehemals einzelne Pflanzungen von Pappeln und Weiden vorgenommen. Den Rest konnte die Natur selbst besorgen, weil das Gebiet für Massentourismus durch weiträumige Absperrungen für den Erholungstourismus nicht zugänglich war. Heute, nach einigen Jahrzehnten, hat sich zwar "nur" ein Ersatzbiotop gebildet, welches aber einer natürlichen Weichholzaue sehr nahe kommt. Derartige Renaturierungsmaßnahmen sollten in viel größerem Umfang praktiziert werden.

Ruderalfloren, früher in und um die Dörfer, an Wegen, Dämmen und anderswo weitverbreitet, haben vielerorts wegen chemischer "Unkraut"Bekämpfung keine Bleibe mehr. Sie finden sich daher heute vorwiegend in Refugien wie verlassenen Gehöften, am Rande von Müllplätzen oder in ehemaligen Abbaustellen. Hier sollten sie geduldet und gefördert werden, auch wenn sie sich dank der "Bracheprogramme" in vielen Bundesländern jüngst wieder in die Feldmark hinaus bewegen.

Trockenrasen: Nährstoffarme Böden, auf denen Sandmagerrasen, Mauerpfeffer-Pionierfluren u. a. m.

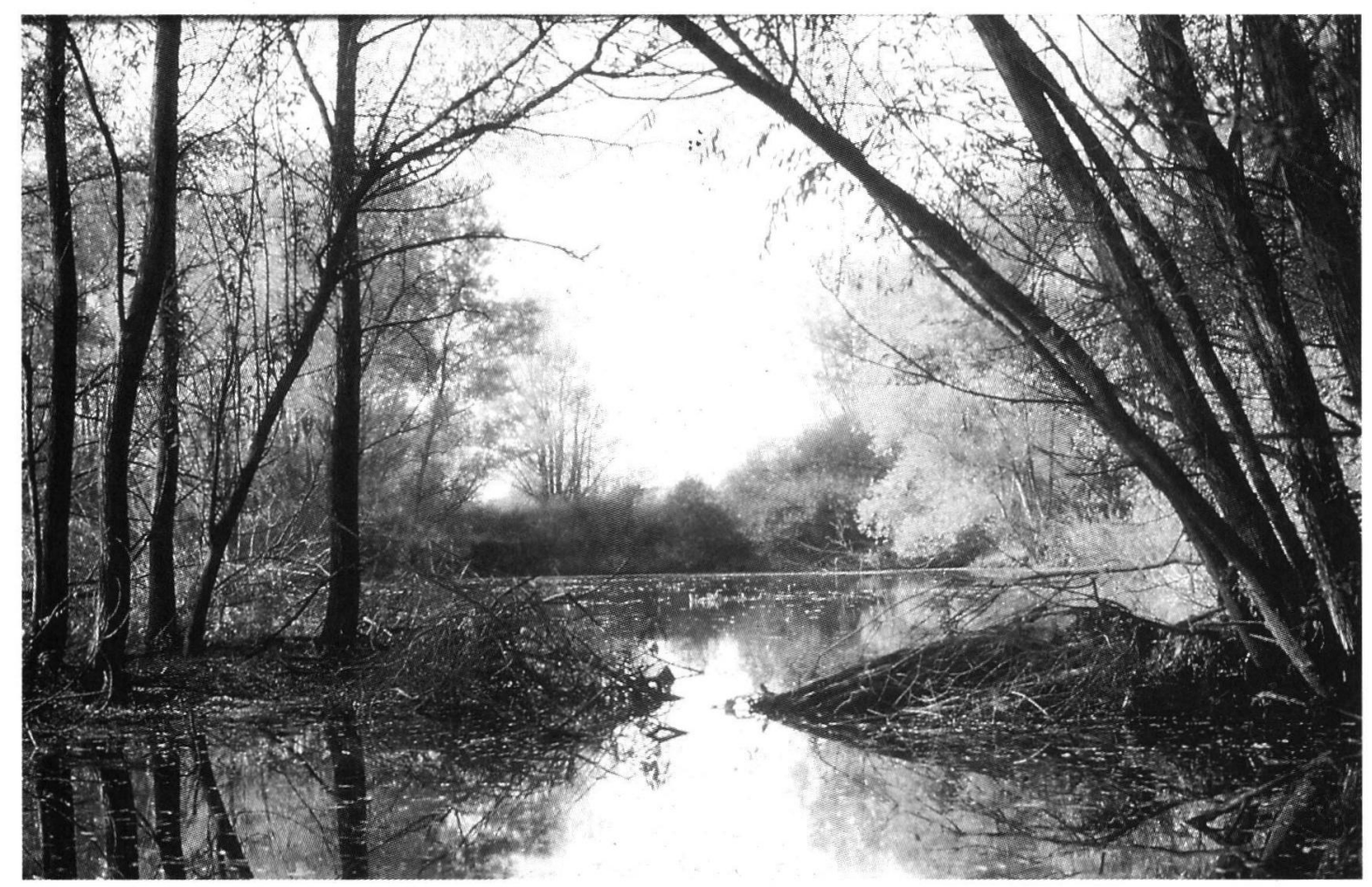

Abb. 6: Weichholzauenwald in renaturierter Kiesgrube 
wachsen und bodenbewohnende Insekten nisten können, sind in unserer fast überall gedüngten Landschaft selten geworden. Sand- und Kiesgruben können dafür ideale Rückzugsstandorte sein.

Biotope für Schmetterlinge, Bienen und andere Insekten: Blütenreiche Wiesen, artenreiche Gebüsche, Frühblüher für Bienen, Nahrungspflanzen für Schmetterlingsraupen u. a. m. können renaturierte Gruben, wenn sie und das umgebende Areal für größere Staudenfluren ausreichend sind (ab ca. 5 ha), in hervorragender Weise bieten.

Amphibienbiotope: Flache, schnell erwärmbare Gewässer/Pfützen mit ihrem Umland sind Laichplätze und Kaulquappen-Kinderstuben, zumal wenn sie kaum oder keinen Anschluß an Fischgewässer haben (Fische fressen Laich, Kaulquappen und z. T. auch ausgewachsene Molche). Manche Amphibienarten leben auf dem feuchten Land, andere im Wasser und wieder andere verlassen als erwachsene Tiere das feuchte Element. Bewachsene Flachwasserzonen und offene, sandig-steinige Uferbereiche mit Geröll- und Schutthaufen werden als Winterquartier bevorzugt. Viele Amphibien konnten nur in renaturierten, "nicht oder nur leicht aufgeräumten" Abbaustellen überleben. Wir haben die Chance, auch hier mit sehr einfachen Mitteln ihr Überleben zu gewährleisten.

\section{Biotopentwicklung und Pflegemaßnahmen}

Von besonderer Bedeutung ist stets die Abstimmung zwischen Abbaubetrieben und Naturschutzbehörden über die Fragen, wie "aufgeräumt" eine Grube zurückgegeben werden muß, wieviel Zeit der Natur zur Eigenentwicklung zu geben ist oder wie ungeduldig der Naturschutz auf "schöne" Flächen oder auf ökologisch wertvolle Areale zu warten hat.

Bei allen Planungen seitens oder für den Naturschutz muß man sich darüber im klaren sein, daß fast jede Widmung einer Fläche für den Naturschutz auch entsprechende Pflegemaßnahmen erfordert, wenn man das angestrebte Naturschutzziel, z. B. Magerrasen, Heidefläche oder Flachwasserzone mit Röhrichtinseln auch auf Dauer erhalten will. Anderenfalls würde unweigerlich auf fast allen Flächen, außer auf natürlichem Hochmoor, Watt/Vorland und aktiver Düne, ein Durchlaufen verschiedener natürlicher Entwicklungsstadien von der Krautschicht über das Buschstadium bis hin zum Wald als Endstadium nach vielen Jahrzehnten zu beobachten sein. Die Entscheidung ob, wann und wie weitgehend $\mathrm{zu}$ irgendeiner Zeit in die natürliche Sukzession eingegriffen werden soll oder darf, grenzt fast an ein Glaubensbekenntnis. Hier wäre mehr pragmatisches Vorgehen vonnöten. Will man einen bestimmten Biotop erhalten und pflegen, dann bedeutet dies unweigerlich, daß irgendwann ein anderer Teil des sich in ständiger Fortentwicklung befindlichen Ökosystems, gewiß ebenso erhaltenswert wie der primär unter Schutz gestellte Biotop, zugunsten des ersteren vernichtet werden muß. Will man dies nicht, wird die Natur sich ihr Recht nehmen, indem ein Biotop durch ein anderes Sukzessionsstadium ersetzt, langsam zurückgedrängt wird. Dies ist Natur!

\section{Forderungen}

Gut geplante Renaturierung kann unbestritten zur Biotopvielfalt und zur Biotopvernetzung beitragen. Damit wird dies zu einem wichtigen Hilfsmittel eines modernen, in die Zukunft gerichteten Naturschutzdenkens und -handelns. Die Erkenntnis ist gewachsen, daß "Sich selbst überlassen", "Nichtstun als Naturschutz", "Mut zur Wildnis und Unordnung" oft ein besserer Weg hin zu ökologisch verträglicher Wiedereingliederung einer Abbaustelle ist als eine allzu tatkräftige Hilfe (= Rekultivierung) dabei. Dieses progressive und kreative Naturschutzdenken ist auf dem Vormarsch und wird irgendwann auch in gesetzlicher Form niedergeschrieben. Je eher man beginnt, selbst Erfahrung damit zu sammeln und auszuwerten, desto besser können jene, die die entsprechenden Richtlinien und Verordnungen erarbeiten, mit wirklichkeitsnahen und auch realisierbaren Vorschlägen unterstützt werden. Die Steine- und Erden-Industrie muß in ihrem Handeln und Denken der Gesetzgebung ein gutes Stück vorauseilen und entsprechende Politikberatung betreiben, damit sich künftige Gesetze, Verordnungen etc. nicht gegen sie entwickeln werden.

Zum Schluß seien einige Forderungen zur Diskussion gestellt:

- Die Beeinflussung des Landschaftsbildes durch den Abbau oberflächennaher Rohstoffe sollte nicht nur in lokalen Einzelstudien, sondern vermehrt und vertiefend auf ihre regionale und überregionale Bedeutung hin untersucht werden. Dabei sollten u. a. auch geomorphologische und landschaftsästhetische Aspekte aufgegriffen werden. Die Ergebnissse sollten wissenschaftlich sauber begründet, nachvollziehbar und für Nichtwissenschaftler verständlich dargelegt werden.

- Die Entwicklung von Fauna und Flora sollte in geeigneten und für bestimmte Landschaftsteile typischen Abbaugebieten vor, während und nach der Rohstoffgewinnung wissenschaftlich sauber erfaßt, dokumentiert und bekanntgegeben werden. Diese Untersuchungen 
sollten ein breites Spektrum an Faunen- und Florenelementen abdecken, nicht nur die vielerorts studierten Vögel, Amphibien, Laufkäfer, Orchideen, Enziane usw.

- Praktiker sollten ihre Erfahrungen über erfolgreiche Renaturierungsmaßnahmen in geeigneter Form bekannt machen.

- Behörden, Wissenschaft und Industrie sollten mehr als bisher gemeinsam in geeigneten Abbaugebieten Maßnahmen des Biotop- und Artenschutzes durchführen. Für Unternehmer sind Risiken und Kosten der Renaturierung zumeist durchaus mit denen herkömmlicher Rekultivierungsmaßnahmen vergleichbar.

- Abbauverfahren, die eine natur- und landschaftsgerechte Wiederherrichtung erleichtern, müssen erprobt und zur technischen Routine entwickelt werden.

Im Rahmen der vorliegenden Ausführungen können aus dem breiten Feld der Renaturierung von
Sand- und Kiesgruben nur ausgewählte Aspekte behandelt werden. Die Darlegungen sollen vor allem dazu anregen, sich vertiefend mit diesen Problemen zu beschäftigen.

\section{Schriftenverzeichnis}

Deutscher Bund für Vogelschutz - Landesverband BadenWürtTEMBERG e. V. (1981): Lebensraum Kiesgrube, 25 S., 68 Abb., 1 Tab.; Basel.

Stein, V. (1985): Anleitung zur Rekultivierung von Steinbrüchen und Gruben der Steine- und Erden-Industrie. - 127 S., 9 Abb., 2 Tab.; Köln (Deutscher InstitutsVerlag).

Geringfügig geänderte Fassung eines Vortrages, gehalten auf der Fachtagung "Produktion von Sand und Kies" des Bundesverbandes der Deutschen Kies- und Sandindustrie e. V. und des Lehrstuhls für Aufbereitung, Veredlung und Entsorgung der RWTH Aachen, 19. - 20. 02. 1992, Aachen.

Manuskript eingegangen am 30. 9. 1992 